J. Clin. Chem. Clin. Biochem.

Vol. 16, 1978, pp. 543-545

\title{
Zur diagnostischen Aussagekraft der Lipoprotein-X-Bestimmung
}

\author{
Von $R$. Wepler
}

Medizinisch-klinisches Labor des Bundeswehrkrankenhauses Ulm (Chefarzt: OTA Dr. Borkowski) und

\section{K. Rommel}

Department für Klinische Chemie des Zentrums für Interdiszịplinäre Medizinische Einheiten der Universität Ulm

(Eingegangen am 27. Januar/16. Juni 1978)

Zusammenfassung: Bei 221 Patienten mit und ohne Cholestase wurde LP.X im Serum untersucht. Die diagnostische Empfindlichkeit und die diagnostische Spezifität des Tests wurden mit 0,9 bzw. 0,88 errechnet. Bei Anwendung dieses Tests in einem nichtselektionierten Kollektiv ist der prädiktive Wert des positiven Tests jedoch sehr gering, während derjenige des negativen Tests sehr groß wird. Für die diagnostische Aussage bedeutet dies, daß LP-X in der Praxis besser zum Ausschluß als zum Nachweis einer Cholestase geeignet ist.

\section{Diagnostic value of the lipoprotein-X determination}

Summary: LP-X was investigated in the serum of 221 patients with and without cholestasis. The diagnostic sensitivity and the diagnostic specificity of the test were 0.9 and 0.88 , respectively. When this test is used on a non-selected collective, however, the predictive value of the positive test is very low, whereas negative results have a high diagnostic value. Thus, in practice, LP-X is more suitable for the exclusion, rather than the detection of cholestasis.

\section{Einleitung}

Das seit wenigen Jahren für die Diagnostik der Cholestase vorgeschlagene Lipoprotein X (LP-X) wurde hinsichtlich seiner diagnostischen Aussage bisher von allen Untersuchern positiv beurteilt (1-5). Dabei ist zu berücksichtigen, daß bei etwa $50 \%$ der Neugeborenen LP-X in den ersten Lebenswochen im Serum nachgewiesen werden kann, ohne daß klinisch-chemisch eine Cholestase besteht (6). Der Test wird mithin erst jenseits des ersten Lebensjahres als cholestasespezifisch angesehen. Dịe diagnostische Zuverlässigkeit wird allerdings in keiner der erwähnten Publikationen qüantifiżiert. Es blieb bei diesen Untersuchungen ferner unberücksichtigt, daß die Ergebnissse an ausgesuchten Kollektiven gewonnen wurden, woraus nicht ohne weiteres auf die Brauchbarkeit des Tests in unselektierten Kollektiven in der Praxis rückgeschlossen werden darf.

In der vorliegenden Arbeit wird erneut an einem größeren, ausgewählten Kollektiv die Bestimmung von LP-X. durchgefuhrt; die Ergebnisse werden mit dem histologischen Befund verglichenen. Darüber hinaus wird versucht, aus den gewonnenen Daten und den Daten der Literatur Aussagen über die diagnostische Empfindlichkeit und die diagnostische Spezifität des Tests sowie über den prädiktiven Wert eines positiven oder negativen Testausfalls zu gewinnen.

\section{Methodik}

Die Untersuchungen wurden mit dem Niedervolt-Elektrophoresesystem "Rapidophor" Firma Immuno AG, Wien, mit den Reagenzien der gleichen Firma nach deren Arbeitsvorschrift durchgeführt.

Blut von 221 Patienten, bei denen aus diagnostischen Gründen eine Leberblindpunktion gerade durchgeführt worden war oder unmittelbar bevorstand, wurde nüchtern gewonnen und das Serum am Entnahmetag untersucht.

Die histologische Diagnose einer intrahepatischen Cholestase wurde gestellt beim Vorliegen von kleinkalibrigen Gallezylindern in den Leberläppchenzentren. Dabei konnten auch Gallezylinder größeren Kalibers in der Läppchenperipherie vorkommen. Die Periportalfelder und das Sternzellsystem waren unverändert.

Die Ergebnisse der LP-X-Untersuchung und der histologischen Leberuntersuchung wurden in Form einer Vierfeldertafel zusammengefaßt. Folgende Kenngrößen (vergleiche Übersicht bei Büttner (7)) wurden errechnet:

\section{Diagnostische Empfindlichkeit}

Sie bezeichnet die bedingte Wahrscheinlichkeit, daß der Test T (LP-X) positiv ist, wenn die Krankheit K (Cholestase) vorhan- 
den ist, also $P(T / K)$. Sie errechnet sich aus der Häufigkeit des positiven Testausfalls bei Kranken:

$$
P(T / K)=\frac{T K}{T K+\overline{T K}} .
$$

\section{Diagnostische Spezifität}

Sie bezeichnet die bedingte Wahrscheinlichkeit, daß der Test negativ ist $(\overline{\mathrm{T}})$, wenn die Krankheit nicht vorhanden ist $(\overline{\mathrm{K}})$, also $\mathrm{P}(\overline{\mathrm{T}} / \overline{\mathrm{K}})$. Sie errechnet sich aus der Häufigkeit des negativen Testausfalls bei Gesunden:

$$
P(\bar{T} / \bar{K})=\frac{\overline{T K}}{T \bar{K}+\overline{T K}} .
$$

\section{Prävalenz der Erkrankung}

Sie bezeichnet die Wahrscheinlichkeit, daß der Proband eines Kollektivs die Erkrankung hat $(\mathrm{P}(\mathrm{K}))$ und errechnet sich aus der Häufigkeit der Kranken im Gesamtkollektiv:

$$
P(K)=\frac{K}{K+\bar{K}} \text {. }
$$

\section{Prädiktiver Wert für das positive und das negative Testergebnis} Der prädiktive Wert ist die Wahrscheinlichkeit, daß die Krankheit vorhanden ist, wenn der Test positiv, bzw. daß die Krankheit nicht vorhanden ist, wenn der Test negativ ist, also $P(K / T)$ für den positiven und $\mathrm{P}(\overline{\mathrm{K}} / \overline{\mathrm{T}})$ für den negativen Test. Der prädiktive Wert errechnet sich aus der Häufigkeit der Erkrankung bei positivem Testausfall:

$$
P(K / T)=\frac{T K}{T K+T \bar{K}}
$$

bzw. aus der Häufigkeit der Abwesenheit der Erkrankung bei negativem Testausfall:

$$
\mathrm{P}(\overline{\mathrm{K}} / \overline{\mathrm{T}})=\frac{\overline{\mathrm{TK}}}{\overline{\mathrm{T}} \mathrm{K}+\overline{\mathrm{TK}}} .
$$

\section{Ergebnisse und Diskussion}

Von insgesamt 221 Patienten hatten 102 im histologischen Bild eine Cholestase (K). Bei allen diesen Patienten war LP-X positiv (T). 119 Patienten hatten histologisch keine Cholestase $(\overline{\mathrm{K}})$, davon war bei 101 Patienten LP-X negativ $(\bar{T})$ und bei 18 Patienten war es falsch-positiv. Dicse Ergebnisse sind in Abbildung 1 in Form einer Matrix zusammengefaßt. Daraus errechnet sich nach den im methodischen Teil angegebenen Formeln eine diagnostische Empfindlichkeit des Tests von 1,0 und eine diagnostische Spezifität von 0,84 . Als bedingte
Abb. 1. Ergebnismatrix der LP-X-Untersuchung an 221 Personen.

\begin{tabular}{lll}
\hline & $\mathrm{K}$ & $\overline{\mathrm{K}}$ \\
\hline $\mathrm{T}$ & 102 & 18 \\
& $=$ richtig positiv & $=$ falsch positiv \\
$\overline{\mathrm{T}}$ & 0 & 101 \\
& $=$ falsch negativ & $=$ richtig negativ \\
\hline & 102 Kranke & 119 Gesunde \\
\hline
\end{tabular}

Wahrșcheinlichkeit formuliert bedeutet dies, daß die Wahrscheinlichkeit, daß bei einer Cholestase LP-X positiv ist, gleich $1,0(100 \%)$ ist, während die Wahrscheinlichkeit, daß bei Gesunden LP-X negativ ist, gleich 0,84 $(84 \%)$ beträgt. Die Prävalenz in unserem Kollektiv $(\mathrm{P}(\mathrm{K}))$ war 0,46 , d. h. $46 \%$ der untersuchten Patienten hatten eine Cholestase.

Werden entsprechende Berechnungen aus den Ergebnissen anderer Autoren angestellt, bei denen die Cholestase ebenfalls histologisch verifiziert oder ausgeschlossen wurde, so finden sich sehr gute Übereinstimmungen mit unseren Ergebnissen, wie in Tabelle 1 dargestellt. Die diagnostische Empfindlichkeit wird zwischen 0,82 und 1,00 , die diagnostische Spezifität zwischen 0,85 und 0,99 errechnet. Diese beiden Kenngrọß gig vom untersuchten Kollektiv, daher ist der direkte Vergleich zulässig.

Die prädiktiven Werte sind von der Prävalenz der Erkrankung abhängig (8): beträgt der prädiktive Wert für den positiven Test bei der in unserem Kollektiv vorliegenden Prävalenz von 0,46 noch $92 \%$, so sinkt er in einem unselektionierten Kollektiv, bei dem die Prävalenz auf Werte unter $1 \%$ abșinken kann, unter $12 \%$. Bei einer Screening-Untersuchung, wo niedrige Prävalenzen zu erwarten sind, wäre ein positives Testergebnis praktisch ohne diagnostische Aussagekraft. Das Einsatzfeld des qualitativen LP-X-Nachweises ist damit ein hochselektioniertes Kollektiv.

Da der prädiktive Wert für das negative Testergebnis mit abnehmender Prävalenz kleiner wird, ist in Kollektiven mịt niedriger Prạ̈valenz der Test geeignet, eine Cholestase auszuschließen.

Aus diesen Ergebnissen wird gefolgert, daß die fachgerechte Befundung eines klinisch-chemischen Analysen-

Tab. 1. Diagnostische Kenngrößen, errechnet aus Angaben der Literatur.

Nur die Fälle sind berücksichtigt, bei denen die Diagnose histologisch kontrolliert war.

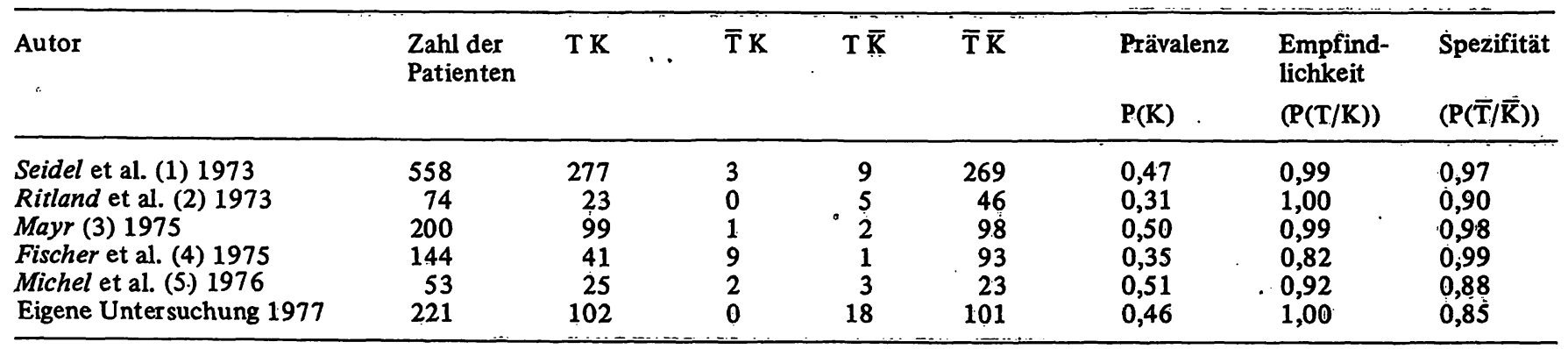


ergebnisses neben der analytischen Beurteilung die Kenntnis von diagnostischer Empfindlichkeit, diagnostischer Spezifität und Prävalenz voraussetzt. Da die Prävalenz in verschiedenen Einsatzfeldern (z. B. gastroenterologische Klinik, Allgemeinklinik, Praxis des niedergelassenen Arztes) unterschiedlich ist, ist auch der prädiktive
Wert unterschiedlich. In einer Screening-Untersuchung mit niedriger Prävalenz erlaubt das negative Testergebnis eher den Ausschluß der Cholestase, während in selektionierten Kollektiven das positive Testergebnis eher zum Nachweis der Cholestase geeignet ist.

\section{Literatur}

1. Seidel, D., Gretz, H. \& Ruppert, C. (1973), Clin. Chem. 19, 86-91.

2. Ritland, S., Blomhoff, J. P., Elgjo, K. \& Gjone, E. (1972), Scand. J. Gastroenterol. 8, 155-160.

3. Mayr, K. (1975), Dtsch. Med. Wochenschr. 100, 2193-2197.

4. Fischer, M., Falkensammer, Ch., Barouch, G., Wuketich, St., Kronsberger, O. \& Schnack, H. (1975), Wien. Klin. Wochenschr. 87, 524-531.

5. Michel, B. \& Ritter, U. (1976), Z. Gastroenterol. 14, 556564.

6. Witt, J. \& Ober, M. (1976), J. Clin. Chem. Clin. Biochem. 14, 197-202.

7. Büttner, J. (1977), J. Clin. Chem. Clin. Biochem. 15, $1-12$.

8. Vecchio, Th. D. (1966), New Engl. J. Med. 274, 1171-1173.

Dr. med. R. Wepler Medizinisch-klinisches Labor Bundeswehrkrankenhaus Ulm Mähringer Weg 105/I D-7900 Ulm 


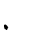

